\title{
ULTRASTRUCTURAL CHANGES IN THE ROGOR INDUCED TOXICITY AND ITS ANTIDOTE ON THE LIVER OF MICE
}

\author{
Archana Prasad ${ }^{*}$ \\ Ashmi Verma
}

\begin{abstract}
Pesticides such as Rogor, Endosulfan, DDT, Daminozole etc. are commonly used to meet the enormous demand of fodder crops. These exert adverse effects at both biochemical and cellular level. They cause several chronic diseases like skin diseases, cancer, liver problems, neural and hormonal diseases. Gross symptoms of damages are seen in very late stages. Thus, present investigation was undertaken to evaluate ultrastructural changes under Scanning Electron Microscope (SEM).

Mice exposed to 2 ppm Rogor for 7 days and 14 days showed behavioural changes temporarily. Ultrastructural observation showed cellular alterations and various damage like blebbing, protuberances, ruffling, lobopodia. Serum SGPT was more in 2 ppm Rogor than those in 2 ppm Rogor along with 3 ppm Selenium treated mice. Selenium treated liver of mice showed striking results with the reduction in toxicities as they are found in 2 ppm Rogor treated liver cells.
\end{abstract}

Key words: Organochlorine, Rogor, Selenium, Blebs, Protuberances.

INTRODUCTION

Use of pesticides has become prevalent not only in farms but also in gardens which has slowly and steadily entered the environment with major contribution in environmental pollution (Dalela et al., 1978; Konar, 1981). Nonbiodegradable pesticides includes organochlorines of which use has been banned but are still been used in Nepal like endosulfan, rogor, etc. EPA suggested that a number of pesticides could cause cancer in people through residues left on food. Nath (2007) reported endosulfan manifested testicular toxicity.

Murray, et al. (1994) published the role of the liver in protecting the organism from dreadful chemicals and its biotransformation to other forms that may not be necessarily toxic.

Though enormous work has been accomplished regarding selenium on the liver of mice but less is known on reduction of pesticide induced toxicity. Hence the present investigation is based on the liver abnormality induced due to a potentially lethal pesticide, Rogor which is nowadays in regular use by ultrastructural study.

\section{MATERIALS AND METHODS}

Five pairs of mice were subjected to acclimatize to the laboratory condition for a week. Each pair (P1-generation) was kept separately in a small cages and was fed on Gulmohar diet. Pregnant females were then transferred to big cage to give birth. After couple of weeks P1-generation were subjected to again breed in small cages. Repeating a colony of mice was formed. Adult mice were then selected for experimental dosage. First phase of investigation was based on pesticide treatment; second phase dealt with antidote treatment. Mice were grouped under four sets with 20 mice each,

Dr. Prasad is a Lecturer Central Department of Zoology, T.U., Kirtipur, Nepal. 
Group A was Normal without any treatment; Group B was Controlled with distilled water; Group C was treated with 2 ppm Rogor for fourteen days; Group D treated with 2ppm Rogor and 3ppm selenium for fourteen days.

Commercial grade Rogor used for investigation is an oily transparent liquid, which gives milky exudation with distilled water. Selenium was obtained in the form of tablets which when dissolved in water formed suspension. Test animals were orally treated and then sacrificed for further processes. For (SEM) studies, the tissues were fixed in $2.5 \%$ gluteraldehyde in $0.1 \mathrm{M}$ chilled $\left(4^{\circ}\right)$ phosphate buffer and $1 \%$ osmic acid in $0.1 \mathrm{M}$ chilled $\left(4^{\circ}\right)$ phosphate buffer.

\section{RESULTS}

Normal liver cell surface appears to posses required cytopodians like microvilli $(\mathrm{Mv})$ responsible to perform certain tissue specific functions like absorption and storage. Framework of blood vessel is well illustrated (Fig. 1).

In seven days rogor treated mice, ruffles were observed (Fig.2). Blebs that usually occur under some stress on the cell surface are predominantly present all over the surface. Macrophages, fenestrations and some spiked structure of irregular shape are found inhabiting the liver cell surface (Fig. 2).

In seven days rogor treatment blebs were more throughout the cell surface with no apparent differences to that of macrophages (Fig. 3). Lobopodia the modified blebs were seen at some places. Microvilli was observed as cloudy appearance with dissolution of its actual organization. Microtrabecular lattices were found, contributing a main constituent to ruffles. Sinusoidal region with fenestrations or cleft near the are seen around the bunch of red blood cells (Fig .3).

With the administration of 3ppm selenium and 2 ppm rogor for seven days showed negligible blebs. Microvilli and filapodias were seen with normal architectures attached to the cell surface. Framework of blood vessels was well organized (Fig. 4).

Fourteen days treatment with selenium and rogor showed highly reduced blebs,ruffles and fenestrations. Clustering of cells is also not found even after fourteen days of treatment of rogor if selenium is administered simultaneously. Microvilli and filapodia with well defined shape were observed (Fig. 5).

Biochemical assay reveals that in the fourteen days treatment of Rogor the value of SGPT was highly increased which indicates the cell to have hepatitis. But in Selenium and Rogor treated mice SGPT value was normal 21.7 unit $/ \mathrm{ml}$. The normal range is $2-24 \mathrm{unit} / \mathrm{ml}$ in mice.

\section{DISCUSSION}

Alterations in the cell surface reflect hormonal imbalance (Flickinger et al., 1979). De Robertis and De Robertis (1970), reported blebs like structure in abnormal adenovirous cells similar to those found in $2 \mathrm{ppm}$ rogor treated. Verma, (1996) reported that the protuberances are found to overcome stress. Sausage shaped cytopodia reffered as lobopodia near microvilli are elongated blebs (Albrechet-Buehler, 1976). From the findings of Cook (1975) and Richardson et al., (1975) it is clear that malignant cell surface membrane changes involve the loss of glycosphingolipids. These alterations are due to reduction of one or more glycosyltransferases present in golgicomplex.

In the present study malignancy of the cell surface is very difficult to report as it is based on short term treatment and such enzymes fractionation are not accomplished. 
Selenium has been established as an integral part against liver degeneration, which is ubiquitous in the present finding (Schamberger, 1983; Schwarz, 1957).

Ruffles or lamellipodia also reflects the hyper activities of the cell and changes in their normal contituients (Flickinger, 1979). Filopadia as a slender structure participate in cell spreading (Ivanova et al., 1976) and act as a tactile sensory investigation of the environment (Gey, 1955; Albrechet-Buehler, 1977).

With the selenium treatment ameliorating effect was seen just after seven days treatment. Present study supports the reports revealing the toxicities induced due to pesticide and helps us to be aware of the fact that we are definitely getting exposed to the deadly chemicals which shows symptom after being accumulated in the cells of our body.

\section{WORKS CITED}

Albrecht-Buehler, G. (1976). Filopodia of spreading 3T3 cells: do they have a substrate exploring function? J. Cell Biology; 69:275-286.

Albrecht-Buehler, G. (1977). Daughter 3T3 cells. Are they mirror images of each other? J. Cell Biology; 72,595-603.

Cook, J.A. (1975). The golgi apparatus. Vol. 77; Oxford University Press.

Dalela,R.C., Bhatanagar, M.C., Tyagi, A.K and Verma, S.R. (1978). Histological damage of gills in Channa gauchua after acute and subacute exposure to Endosulfan and Rogor. Microskopic (wein). 35:301-307.

De Roberties, E.D.P. and De Roberties E.M.F. (1970). Cell and molecular biology. INFO-MED. International edition.

Flickinger, C.J., Katchai, H.C., Brown, J.C., Ogilive, J.W. (1979). Medical cell biology. Holt Saunders International Journal.

Gey, G.O. (1955). Some aspects of the constitution and behaviour of normal and malignant cells maintained in continuous culture. In Harvey Lect. Academic Press. New York, pp. 154-338.

Ivanova, O.Y., Margolis, L.B., Vasiliev, J.M., Gelfand, I.M. (1976). Effect of colcmid on the spreading of fibroblasts in culture. Exp. Cell Res.; 101, 207-219.

Konar, S.K. (1981). Pollution of water by pesticides and its influences on aquatic ecosystem. India Rev. Life Sci.1; 319-165.

Murray, M. Butler, A.M. (1994). Hepatic biotransformation of parathion: role of cytochrome p. 450 in NADPH and mediated microsomal oxidation in vitro. Chemical research in toxicology. Nov. Vol. 7. No. 6, pp. 792.

Nath,A.(2007).New millennium pose problems for reproductive-healthdue to pesticides: controlled by herbal therapy. Abst. $2^{\text {nd }}$ Bihar Vigyan Congress, pp. 33.

Richardson, C.A..; Baker, S.R.; Moore, D.J. and Keenan, T.W. (1975). "Thegolgi apparatus".Biochem. Biophys. Acta. 417-475.

Schambeger, T.J. (1983). Metabolism of selenium. IN: Biochemistry of Selenium. Frieden plenum, New York. Vol. 2, pp. 59-175.

Schwartz, K. and Foltz, C.M. (1957). Selenium as an integral part of factor III against dietary liver degeneration. J. Am. Chem. Soc. 79. 3292-3293.

Verma, A. (1966). Biochemical and Ultrastructural Changes after Malathion treatment in the ovary of the Heteropneustes fossilis. Patna University, Thesis. 


\section{PLATE I}

Scanning Electron Microphotograph of 2 ppm Rogor and 3 ppm Selenium treated liver of mice

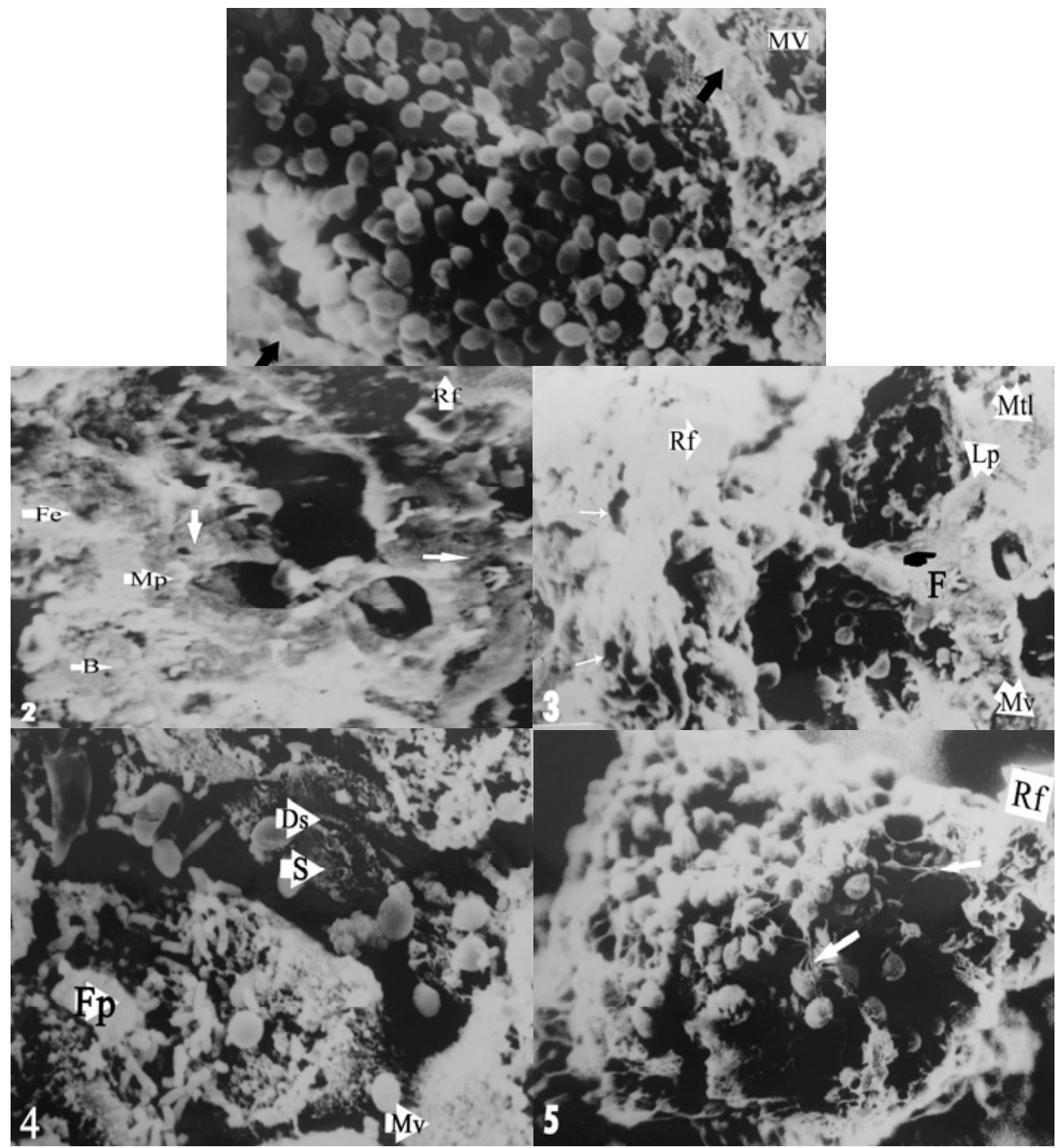

Fig 1: Normal liver: Cell surfaces are normal $(\rightarrow)$.Shape of microvilli $(\mathrm{Mv})$ responsible for the storage of membrane, gives normal appearance to the cell. Sinusoidal region also lies normally along the clustered blood cells. $(\rightarrow)$

Fig 2: Mice treated with 2ppm rogor for seven days. Prominent blebs (B) with macrophages $(\mathrm{Mp})$ and fenestrations ( $\mathrm{Fe}$ ) on the surface: ruffling protrusions (Rf)

Fig 3. Mice treated with $2 \mathrm{ppm}$ rogor for fourteen days: ruffling (Rf) with lobopodias (Lp) as elongated blebs. Microtrabecular lattice (Mtl) main component of ruffles, initiating its formation. Deep fenestrations $(\rightarrow)$ with reduced microvilli and protruded blebs. (F)

Fig 4. Mice treated with $2 \mathrm{ppm}$ rogor and $3 \mathrm{ppm}$ selenium for seven days. Microvilli (Mv) and filopodia (Fp) well defined. Sinusoidal wall (S) and perisinusoidal (Ds) well marked.

Fig 5. Mice treated with $2 \mathrm{ppm}$ rogor and $3 \mathrm{ppm}$ selenium for fourteen days. Ruffles (Rf), blebs and fenestrations reduced. No clustering of cell. Well illustrated network of blood vascular system $(\rightarrow)$. 